\title{
Antimicrobial Suspectibility of Bacterial Isolated from Burn Unit in Dr. Hasan Sadikin General Hospital Bandung, Indonesia from June to September 2013
}

\author{
Yodya Evila, ${ }^{1}$ Sunarjati Sudigdoadi, ${ }^{2}$ Hardisiswo Soedjana ${ }^{3}$ \\ ${ }^{1}$ Faculty of Medicine Universitas Padjadjaran, ${ }^{2}$ Department of Microbiology and Parasitology, \\ Faculty of Medicine Universitas Padjadjaran, ${ }^{3}$ Department of Plastic Surgery Faculty of Medicine \\ Universitas Padjadjaran/Dr. Hasan Sadikin General Hospital Bandung
}

\begin{abstract}
Background: Development of infection in burned patients is a crucial matter because it may increase morbidity and mortality. Factors contributing to high risk for infections are disrupted skin barrier, dysfunction of the immune system and prolonged hospitalization. Crowded hospital condition and transfer of patients from one unit to another can affect development of infection caused by environmental factors. The aim of the study was to identify the antimicrobial suspectibility of bacterial isolated from Burn Unit in Dr. Hasan Sadikin General Hospital, Bandung.

Methods: A descriptive study was carried out in Burn Unit Dr. Hasan Sadikin General Hospital Bandung, Indonesia, from June to September 2013. A total of 30 environment samples were collected and cultured to identify the bacterias. Futhermore, the colonies found, underwent antimicrobial susceptibility test.

Results: Most common bacteria found from the environmental samples in Burn Unit was Staphylococcus saprophyticus (24\%). Other bacterias found were Klebsiella pneumonia (17\%), Acinetobacter baumanii (15\%), Staphylococcus epidermidis (12\%), Bacillus sp.(12\%), Pseudomonas sp.(7\%), Staphylococcus aureus (5\%), Enterobacter aerogenes (5\%) and Serratia sp. (2\%). Some bacteria still had good sensitivity to antibiotics while the Enterobacteriaceae were almost completely resistant to antimicrobial used in the study.

Conclusions: The most common bacteria found from the environment samples in Burn Unit is Staphylococcuss aprophyticus. Additionally, Klebsiella pneumoniae as one of the Enterobacteriaceae groups, appears as an emerging hospital associated infection pathogen with their resistant to many antimicrobials. [AM].2015;2(3):324-31]
\end{abstract}

Keywords: Antibiotic susceptibility, bacteria, burn unit

\section{Introduction}

Hospital associated infection is one of the causes of increased mortality and morbidity in hospitals, and it is an important health burden worldwide. $^{1}$ The most common hospital associated infection is burn wound infection. Out of $64.72 \%$ cases of hospital associated infections in burn patients, $62.02 \%$ of them are burn wound infections. ${ }^{2}$ In burn patients, infections arise from multiple sources such as from the wound itself, decreased immune system, and diagnostic procedures. ${ }^{3,4}$ Burn wound provides a place prone to colonization by opportunistic organisms from either endogenous or cross-infection due to disrupted of the skin barrier. $^{5}$ This crossinfection arises among others by contact from patient to patient, and the environment to the patient. ${ }^{6}$ Crowded condition of the hospital, transfer of patients from one unit to another and concentration of patients which is easy for being infected in one area, can affect development of hospital infections caused by environmental factors. Avni et al 3 stated that septic shock is a major problem among burn patients because it precedes multi organ dysfunction and death.

Based on data from the study in the Burn Unit at Dr. Cipto Mangunkusumo General

Correspondence: Yodya Evila, Faculty of Medicine, Universitas Padjadjaran, Jalan Raya Bandung-Sumedang Km.21, Jatinangor, Sumedang, Indonesia, Phone: +6287823073772 Email: yodyaev@hotmail.com 
Table 1 Bacteria Isolated from the Environment Samples

\begin{tabular}{lcc}
\hline \multicolumn{1}{c}{ Bacteria } & Frequency & $\mathbf{\%}$ \\
\hline Staphylococcus & & \\
S.saprophyticus & 10 & 24.39 \\
S. epidermidis & 5 & 12.20 \\
S. aureus & 2 & 4.87 \\
Enterobacteriaceae & & \\
Klebsiellapneumoniae & 7 & 17.07 \\
Enterobacteraerogenes & 2 & 4.87 \\
Serratia sp. & 1 & 2.44 \\
Non-enterobacteriaceae & & \\
Acinetobacterbaumanii & 6 & 14.63 \\
Bacillus sp. & 5 & 12.20 \\
Pseudomonas sp. & 3 & 7.32 \\
Total & 41 & \\
\hline
\end{tabular}

Hospital, Jakarta in 2010, the bacteria most commonly found in the culture from environmental samples were K. pneumoniae, S. aureus, Enterobacter sp, Acinetobacter sp, Bacillus sp, Pseudomonas aeruginosa and Citrobacter freundill.7 Burn wound infections can be especially difficult to eradicate because of multidrug resistance. Microorganisms that are resistant to antibiotics usually lead to an

increase in considerable morbidity, and health care costs. The objective of this study was to identify the antimicrobial suspectibility of bacterial isolated from the Burn Unit at Dr. Hasan Sadikin General Hospital Bandung.

\section{Methods}

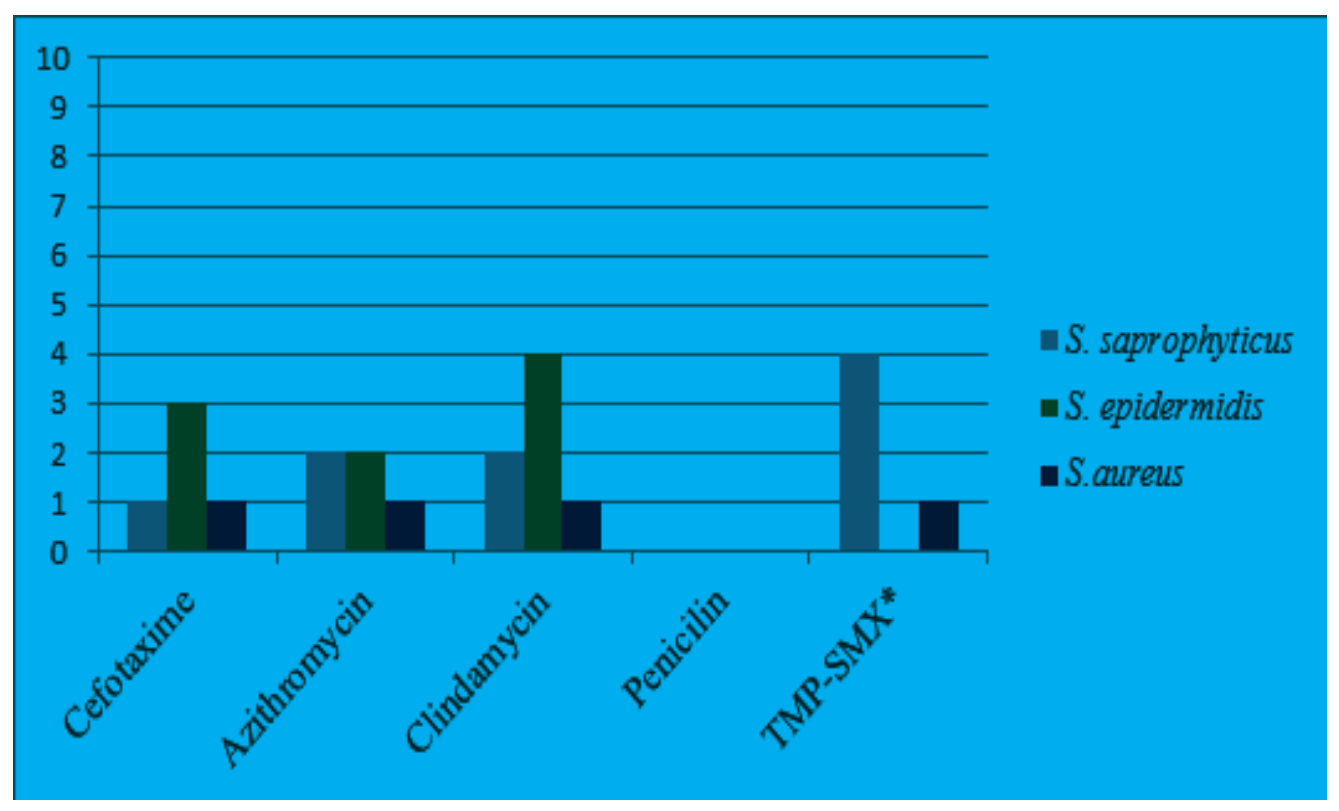

Figure 1 Antibiotic Sensitivity of Staphylococcus

Note: TMP-SMX: Trimetophrim-sulfamethoxazole 
This study was carried out from June to September 2013 in the Burn Unit at Dr. Hasan Sadikin General Hospital Bandung and this study was approved by the Health Research Ethics Committee. Environmental samples were collected from 4 care rooms with $2 \mathrm{~m}$ length and $3 \mathrm{~m}$ width each, a bathroom, nurse station and doctor's room. A total of 30 surface swabs were taken, consisted of 4 samples from the floor, 4 samples from the bed sheet, 4 samples from the bedside table, 4 samples from the curtain, 4 samples from the exhaust fan, 2 samples fromthe air conditioner, 2 samples from the central room door handle, 1 sample from the bathroom door handle, 1 sample from the storage room door handle, 2 samples from the dressing cart, 1 sample from the nurse station and 1 sample from the

\section{Table 2 Distribution per Sampling Sites of Bacteria Isolated from Environment Samples}

\begin{tabular}{|c|c|c|}
\hline Bacteria & Location & Frequency \\
\hline \multicolumn{3}{|l|}{ Staphylococcus } \\
\hline \multirow[t]{5}{*}{ S. saphrophyticus } & Bedside table & 4 \\
\hline & Bed sheet & 2 \\
\hline & Nurse station & 2 \\
\hline & Curtain & 1 \\
\hline & Injection table & 1 \\
\hline \multirow[t]{4}{*}{ S. epidermidis } & Central room door handle & 2 \\
\hline & Bed sheet & 1 \\
\hline & Bedside table & 1 \\
\hline & Dressing car & 1 \\
\hline \multirow[t]{2}{*}{ S.aureus } & Exhaust fan & 1 \\
\hline & Dressing car & 1 \\
\hline \multicolumn{3}{|l|}{ Enterobacteriaceae } \\
\hline \multirow[t]{3}{*}{ K. pneumoniae } & Exhaust fan & 3 \\
\hline & Bed sheet & 1 \\
\hline & Bedside table & 1 \\
\hline \multirow[t]{4}{*}{ Enterobacteraerogenes } & Curtain & 1 \\
\hline & Air conditioner & 1 \\
\hline & Curtain & 1 \\
\hline & Central room door handle & 1 \\
\hline Serratia sp. & Bathroom door handle & 1 \\
\hline \multicolumn{3}{|l|}{ Non-Enterobacteriaceae } \\
\hline \multirow[t]{3}{*}{ A. baumanii } & Floor & 3 \\
\hline & Curtain & 2 \\
\hline & Bed sheet & 1 \\
\hline \multirow[t]{4}{*}{ Bacillus sp. } & Curtain & 2 \\
\hline & Bathroom door handle & 1 \\
\hline & Air conditioner & 1 \\
\hline & Exhaust fan & 1 \\
\hline \multirow[t]{2}{*}{ Pseudomonas sp. } & Floor & 2 \\
\hline & Exhaust fan & 1 \\
\hline
\end{tabular}


injection table. All the samples were collected in the afternoon.

Furthermore, sterile swabs filled with 0.5 $\mathrm{ml}$ of trypticase soy broth (TSB) were used to swab the floor, elevated surface and inanimate objects which were constantly in contact with health care workers and patients.8 All swabs were dipped into TSB as transport medium and were brought and processed in the Microbiology Laboratory Faculty of Medicine Universitas Padjadjaran, Jatinangor. Each sample was inoculated on both blood agar and Mac Conkey agar. All samples were incubated for 24 hours at $37^{\circ} \mathrm{C}$. Then, selected colonies with typical morphology and pigmentation on the blood agar and Mac Conkey agar were Gram stained. Next, the Gram positive coccus which grew on blood agar was then subjected to catalase test and coagulation test. Coagulase negative Staphylococci was identified with novobiocin susceptibility test. The Gram negative rod which grew on Mac Conkey agar then was identified with biochemical test including kligler iron agar (KIA), motility indol urea (MIU) and citrate test. The data were presented as frequency distribution and showed in tables.

The procedure was then preceded to antibiotics susceptibility test which was performed on Mueller-Hinton agar by KirbyBauer and Stokes' disk diffusion method recommended by the Clinical Laboratory and Standard Institute (CLSI). Antibiotics used for the susceptibility testing were based on the group A antibiotics from CLSI which are considered appropriate for inclusion in a routine, primary testing and routine reporting and also cefotaxime which is given as initial treatment for all burn patients. The inhibition zone diameter was then measured and noted according to CLSI 2012 criteria which was specific for each bacterium and antibiotics, and classified as susceptible (S), intermediate (I) and resistant (R). ${ }^{9}$

\section{Results}

This study indicated that Staphylococcus saprophyticus was the most common gram positive bacteria found (24.39\%), and the most common gram negative bacteria found was Klebsiella pneumoniae (17.07\%) (Table 1).

Most common inanimate objects colonized by the bacteria were the curtains, as many as 7 colonies, followed by the bedside table and exhaust fan (6 colonies each) (Table 2).

Additionally, antimicrobial suspectibility tests were carried out to all bacterial colonies isolated in the environmental samples. Figure 1 shows that Staphylococcus were completely resistant to penicillin. Also, the highest number of sensitivity was toward clindamycin.

Figure 2 shows that Enterobacteriaceae were completely resistant to almost all antibiotics tested, only gentamycin had a slight effect to the bacteria.

Bacteria A. baumanii found in this study was still sensitive to meropenem and ampicillin-

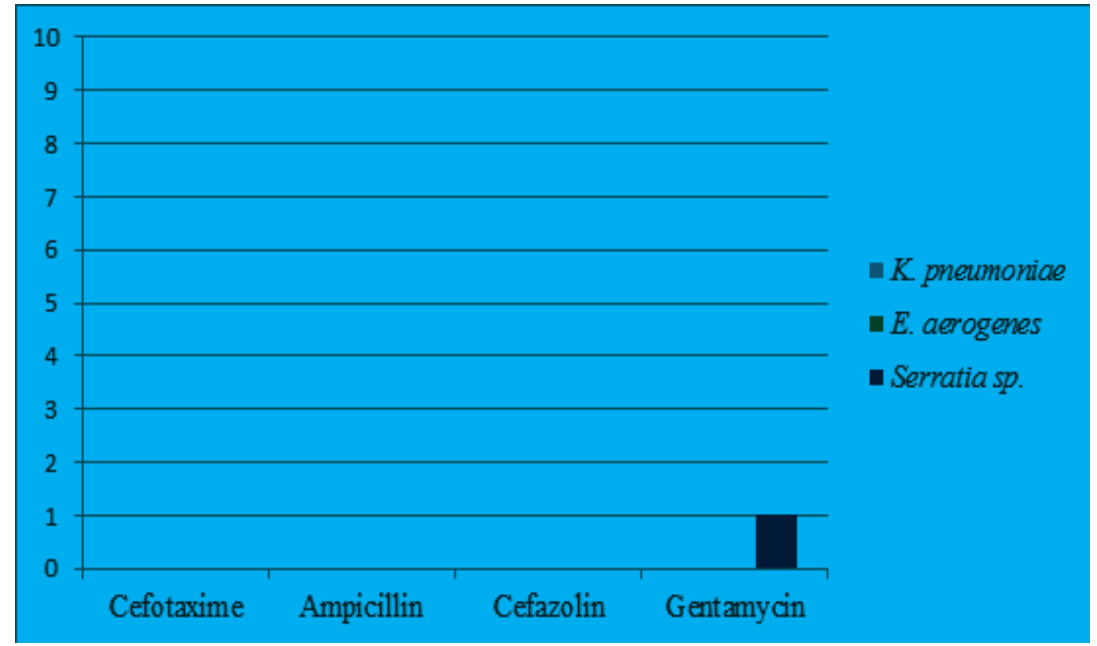

Figure 2 Antibiotic Sensitivity of Enterobacteriaceae 


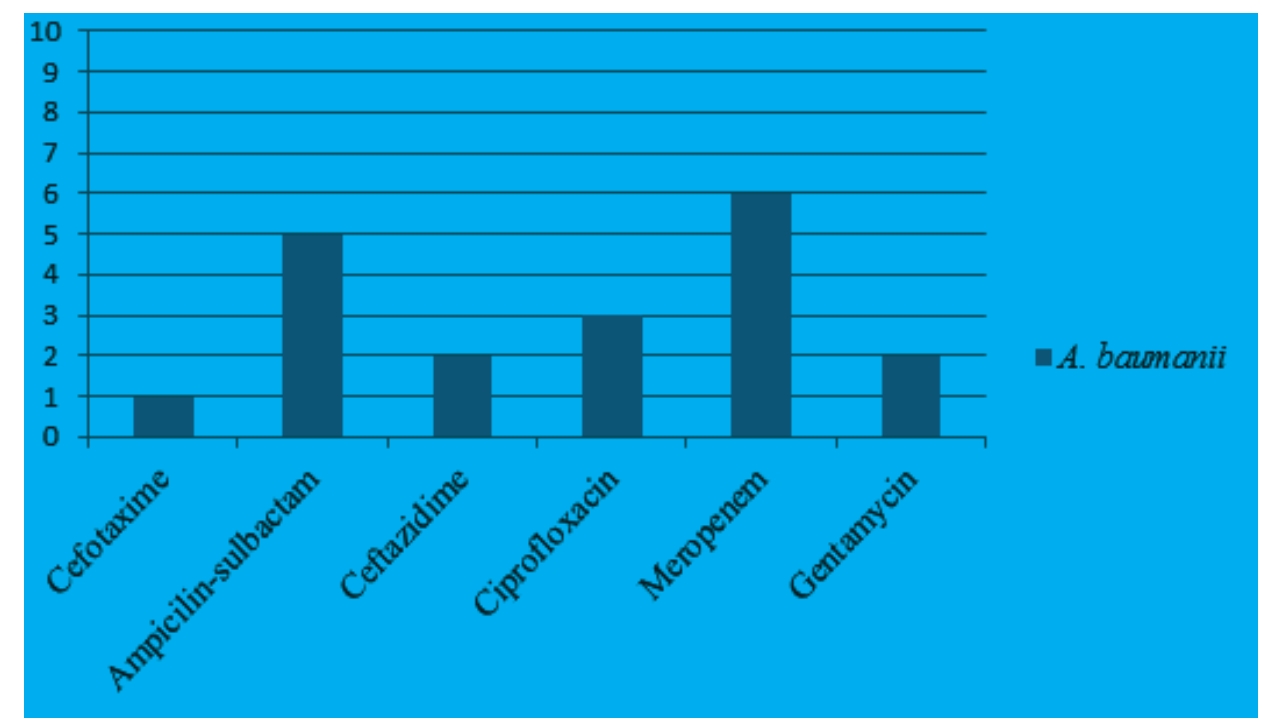

Figure 3 Antibiotic Sensitivity of Acinetobacterbaumanii

sulbactam, but it had least sensitivity to cefotaxime (Figure 3).

\section{Discussion}

Hospital environment provides a place for colonization of microorganisms and some of them were multi resistant to antibiotics. Although health care workers and patients are considered as major sources of hospital infections, however some studies begin to show that the growing role of the environment is also important. ${ }^{10}$ Results of this study indicated that bacteria commonly found in the environment of burn unit were Staphylococcus saprophyticus $(24 \%)$. This is consistent with the study which reported coagulasenegative Staphylococcus as the most predominant organisms in burn infection. ${ }^{5}$ Second, most common bacteria found in this study was Klebsiellapneumoniae (17\%), followed by Acinetobacterbaumannii (15\%).

Compared to the study that had been

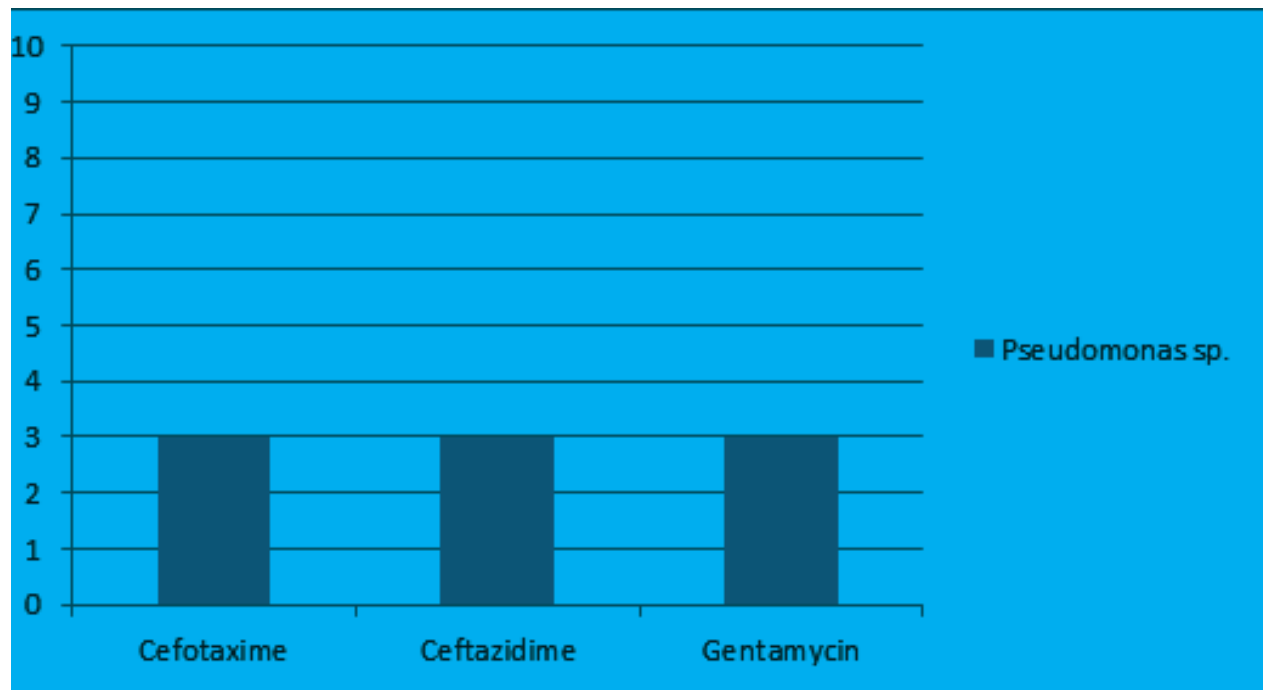

Figure 4 Antibiotic Sensitivity of Pseudomonas sp 
conducted in the Burn Unit at Cipto Mangunkusumo Hospital from January to December in 20107, bacteria found from the environment were K. pneumoniae, S. aureus, Enterobactersp, Acinetobacter sp, Bacillus sp, Pseudomonas aeruginosa and Citrobacter freundill. The results are slightly in contrast with this study even though some bacteria were also found in this study. The differences in the findings of the bacteria between environment of Burn Unit at Dr. Hasan Sadikin General Hospital and Cipto Mangunkusumo Hospital could be possibly due to the geographical factors, condition of the environment including temperature and different hygiene. Another factor affecting the differences could be related to the antimicrobial usage policy. ${ }^{4}$

Besides, Staphylococci are pathogens causing hospital acquired infections and in particular for S. saprophyticus. It is gram positive coccus coagulase negative and common etiology of urinary tract infection. ${ }^{11}$ In this study, S. saprophyticus were most commonly found on the bedside table. Lack of personal hygiene of patients and healthcare workers such as washing hands could cause these bacteria to spread from human to the environment and vice versa. Bacteria S. saprophyticus is not the most common Staphylococcus found in the cultures from blood or wounds of burn patients in the Burn Unit at Dr. Hasan Sadikin General Hospital, which is S. aureus, likely it is because Staphylococcus found in the patient's body are more pathogenic.

Other Staphylococcus found in this study was S. epidermidis (12\%) and S. aureus (5\%). Staphylococcus which is considered as the major pathogen in humans is $\mathrm{S}$. aureus, it is commensal bacteria on the skin, nose, throat and it can survive on dry environmental surfaces. It can be spread through contact with pus from infected sores, skin to skin contact and contact with objects such as towels, bed linen, clothing used by infected patients. ${ }^{11}$ In this study, the susceptibility test of Staphylococcus to antibiotics was performed, and it showed that these bacteria were sensitive to clindamycin, 7 out of 17 colonies were sensitive to clindamycin and fully resistant to penicillin. Mechanism of resistance to penicillin is usually by producing betalactamase enzymes which is under control of plasmid..$^{10}$ Results from this study showed that S. epidermidis were most commonly found on central room door handle and S. aureus were found from exhaust fan and dressing car.

Enterobacteriaceae found in this study were Klebsiellapneumoniae (17\%) as the second most common bacteria found. This result is in accordance with other studies conducted in the Burn Unit at some hospitals in India. ${ }^{12}$ Data from this study showed that $\mathrm{K}$. pneumoniae isolated in this study were most commonly found from exhaust fan. Another Enterobacteriaceae found were Enterobacteraerogenes (5\%) and Serratia sp. $(2 \%)$. As the most common gram negative bacteria found in this study, K. pneumoniae is a pathogen causing hospital infections and is widely available in nature. The main source of infection in the hospital environment by K. pneumoniae are instruments that have been contaminated with the bacteria contact with patients. ${ }^{13}$ It showed resistance to many antibiotics, based on a research conducted in the Neonatal and Pediatric Intensive Care Unit, out of the 130 isolates, $126(97 \%)$ produced extended spectrum beta lactamase (ESBL) and it related to the high mortality rates that occurred..$^{14}$ Although in this study we did not perform the ESBL testing, Enterobacteriaeceae found were subjected to the susceptibility testing and they also showed resistance to almost all antibiotics tested including ampicillin, gentamycin and cephalosporin class antibiotics.

Acinetobacterbaumannii were found as many as six $(15 \%)$ in this study and most commonly isolated from the floors. It is gram negative bacilli and not motile. It is considered as a significant nosocomial pathogen with many infections concentrated in intensive care, burns or high dependency units treating severely ill or debilitated patients. ${ }^{5}$ High degree of resistance to drying and disinfectants, leading to long-term persistence in the hospital environment and to the occurrence of outbreaks of infections affecting immunosuppressant patients such as burn patients and increasing proportion of multidrug resistant isolates make this bacteria become an important problem in burn wound management. Multidrug resistance of Acinetobacter sp. is a cause of global outbreaks. It is probably as a consequence of increasing use of broad-spectrum antibiotics in hospitals. ${ }^{5}$ Results from this study showed that A. baumannii found in the burn unit were fully sensitive to meropenem and still showed high levels of sensitivity to ampicillin-sulbactam, so those antibiotics are still effective to be used for patients in the Burn Unit at Dr. Hasan Sadikin General Hospital.

Pseudomonas sp. found in small amounts in this study were $7 \%$. They are gram-negative 
bacteria widely distributed in soil, water and plants. In this study, these bacteria were most commonly found on the floor. Sometimes, Pseudomonas is an opportunistic pathogen. The main pathogen and much reported as they existed in the hospital environment is $\mathrm{P}$. aeruginosa, however it was not found in this study. Pseudomonas sp. found on environmental swabs in the Burn Unit at Dr. Hasan Sadikin General Hospital showed high levels of sensitivity to all antibiotics tested including the cephalosporin group antibiotics and macrolide group antibiotics.

Another bacteria found from the environment of the burn unit was Bacillus sp. $(12 \%)$. However, Bacillus sp. isolated from the environment in this study did not undergo the susceptibility testing, for it was known that the strain found in the environment is nonpathogenic bacteria.

Bacteria found in the environment were distributed in various inanimate objects, most bacteria colonized the curtains and as many as 7 colonies of bacteria were found there. The number of bacteria colonized on the bedside table and exhaust fan were 6, it made the bedside table and exhaust fan as the second most common objects being colonized by bacteria.

In this study, the swabs were not taken from all environment surfaces of the burn unit because there were limitation of time and fund. In a further study, it might be needed to take swabs from all the surfaces to represent all bacteria colonized the entire environment of burn unit.

This study showed that some bacteria could be ubiquitous in the environment of burn unit. Results from the antibiotics susceptibility test showed that some bacteria still exhibited a high level of sensitivity. The main factors that caused an increase in the prevalence of resistant organisms in hospitals were changes on the organisms causing hospital infections, changes in the organism due to the changes in population characteristics of hospitals, procedures and medical devices used on patients.

Environmental contamination may reach considerable levels and this could result in contamination of the medical instruments, health care workers and patients. Microbiological study and antibiotic susceptibility test from the environment of the burn unit should be conducted routinely to assist in monitoring the type of organisms involved in infections and antimicrobial resistance patterns.
It is important to prevent hospital associated infections caused by environmental factors and reduce contamination and potential cross infection by disinfecting, careful isolation techniques and routine procedures, right antimicrobial choice and good hygiene of the environment, scheduled cleaning of environment in the burn unit using antiseptic solution that can keep infection rates and antimicrobial resistance rates low in burn patients.

\section{References}

1. Peleg, Anton Y, Hooper D. Hospital-acquired infections due to gram negative bacteria. $\mathrm{N}$ Engl J Med. 2010;362(19):1804-13.

2. Chun-cui W. Nosocomial infection in burn unit. Chin Med J. 2006;16(7):22-6.

3. Avni T, Levcovich A, Ad-El DD, Leibovici L, Paul M. Prophylactic antibiotics for burns patients: systematic review and metaanalysis. BMJ. 2010;340:c241.

4. Oncul O, Ulkur E, Acar A, Turhan V, Yeniz E, Karacaer Z, et al. Prospective analysis of nosocomial infections in a burn care unit, Turkey. Indian J Med Res. 2009;130(6):758-64.

5. Septiari BB. Infeksi nosokomial. 1st ed. Yogyakarta: Nuha Medika; 2012. p. 39-49

6. Alaghehbandan R, Azimi L, Lari AR. Nosocomial infections among burn patients in Teheran, Iran: a decade later. Ann Burns Fire Disasters. 2012;25(1):3-7.

7. Wardhana A. Nosocomial infection in burn unit of Cipto Mangunkusumo Hospital, Jakarta. JPR. 2012;1(3):352-6.

8. Rutala W, Katz E, Sherertz R, Sarubbi F. Environmental study of a methicillinresistant Staphylococcus aureus epidemic in a burn unit. J Clin Microbiol. 1983;18(3):683-8.

9. Clinical and Laboratory Standards Institute. Performance standards for antimicrobial susceptibility testing; twenty-second informational supplement. CLSI document M100-S22. Wayne, PA: Clinical and Laboratory Standards Institute; 2012. p. 35-121.

10. DakićI, Morrison D, VukovićD, SavićB, Shittu A, Ježek P, et al. Isolation and molecular characterization of Staphylococcus sciuri in the hospital environment. J Clin Microbiol. 2005;43(6):2782-5.

11. Lowy FD. Staphylococcus aureus infections. N Engl J Med. 1998;339(8):520-32.

12. Srinivasan S, Vartak AM, Patil A, Saldanha $\mathrm{J}$. Bacteriology of the burn wound at the 
Bai Jerbai Wadia Hospital for children, Mumbai, India-a 13-year study, part I-bacteriological profile. Indian J Plast Surg. 2009;42(2):213-8.

13. Podschun R, Pietsch S, Höller C, Ullmann U. Incidence of Klebsiella species in surface waters and their expression of virulence factors. Appl Environ Microbiol.
2001;67(7):3325-7.

14. Bratu S, Landman D, Haag $R$, Recco $R$, Eramo A, Alam M, et al. Rapid spread of carbapenem-resistant Klebsiella pneumoniae in New York City: a new threat to our antibiotic armamentarium. Arch Intern Med. 2005;165(12):1430-5. 